\title{
Thermodynamics of apparent horizon and modified Friedman equations
}

\author{
Ahmad Sheykhi* \\ Department of Physics, Shahid Bahonar University, P.O. Box 76175, Kerman, Iran \\ Research Institute for Astronomy and Astrophysics of Maragha (RIAAM), Maragha, Iran
}

\begin{abstract}
Starting from the first law of thermodynamics, $d E=T_{h} d S_{h}+W d V$, at apparent horizon of a FRW universe, and assuming that the associated entropy with apparent horizon has a quantum corrected relation, $S=\frac{A}{4 G}-\alpha \ln \frac{A}{4 G}+\beta \frac{4 G}{A}$, we derive modified Friedmann equations describing the dynamics of the universe with any spatial curvature. We also examine the time evolution of the total entropy including the quantum corrected entropy associated with the apparent horizon together with the matter field entropy inside the apparent horizon. Our study shows that, with the local equilibrium assumption, the generalized second law of thermodynamics is fulfilled in a region enclosed by the apparent horizon.
\end{abstract}

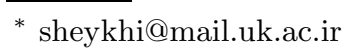




\section{INTRODUCTION}

The pioneer study on the deep connection between gravity and thermodynamics was done by Jacobson [1] who showed that the gravitational Einstein equation can be derived from the relation between the horizon area and entropy, together with the Clausius relation $\delta Q=T \delta S$. Further studies on the connection between gravity and thermodynamics has been investigated in various gravity theories [2 4]. In the cosmological context, attempts to disclose the connection between Einstein gravity and thermodynamics were carried out in [5 9]. It was shown that the differential form of the Friedmann equation in the Friedmann-Robertson-Walker (FRW) universe can be written in the form of the first law of thermodynamics on the apparent horizon. The profound connection provides a thermodynamical interpretation of gravity which makes it interesting to explore the cosmological properties through thermodynamics. Investigations on the deep connection between gravity and thermodynamics have recently been extended to braneworld scenarios [10-12].

It is interesting to note that Friedmann equations, in Einstein's gravity, can be derived by applying the Clausius relation to the apparent horizon of FRW universe, in which entropy is assumed to be proportional to its horizon area, $S=A / 4 G$ [7]. However, this definition for entropy can be modified from the inclusion of quantum effects, motivated from the loop quantum gravity (LQG). The quantum corrections provided to the entropy-area relationship leads to the curvature correction in the Einstein-Hilbert action and vice versa [13]. The corrected entropy takes the form $[14]$

$$
S_{h}=\frac{A}{4 G}-\alpha \ln \frac{A}{4 G}+\beta \frac{4 G}{A},
$$

where $\alpha$ and $\beta$ are positive dimensionless constants of order unity. The exact values of these constants are not yet determined and still an open issue in loop quantum cosmology. These corrections arise in the black hole entropy in LQG due to thermal equilibrium fluctuations and quantum fluctuations [15]. It is important to note that in the literature different kind of modification of entropy expression have studied in classical level for various modified gravity theories [16, 17]. The $\log$ correction to the area-entropy relation appears to have an almost universal status, having been derived from multiple different approaches to the calculation of entropy from counting microscopic states in different quantum gravity models. Let us stress here that although in the literature there is doubt about the second correction term in entropy-corrected relation, however, it is widely believed [14] that the next quantum correction term to black hole entropy have the form $4 G / A$, which leads to the reasonable correction terms to Newton's law of gravitation [18] and will also lead to 
the corrected modified Friedmann equation as we will show in this paper.

Besides, if thermodynamical interpretation of gravity near apparent horizon is generic feature, one needs to verify whether the results may hold not only for more general spacetimes but also for the other principles of thermodynamics, especially for the generalized second law of thermodynamics. The generalized second law of thermodynamics is a universal principle governing the universe. The generalized second law of thermodynamics in the accelerating universe enveloped by the apparent horizon has been studied extensively in [19 21]. For other gravity theories, the generalized second law has also been considered in [22].

The aim of this paper is twofold. The first is to derive modified Friedmann equations by applying the first law of thermodynamics, $d E=T_{h} d S_{h}+W d V$, at apparent horizon of a FRW universe and assuming the apparent horizon has an entropy expression like (1). The other is to see whether the quantum corrected entropy-area relation together with the matter field entropy inside the apparent horizon will satisfy the generalized second law of thermodynamics.

\section{MODIFIED FRIEDMANN EQUATION FROM THE FIRST LAW OF THERMODYNAMICS}

We consider a homogenous and isotropic FRW universe which is described by the line element

$$
d s^{2}=h_{\mu \nu} d x^{\mu} d x^{\nu}+\tilde{r}^{2}\left(d \theta^{2}+\sin ^{2} \theta d \phi^{2}\right)
$$

where $\tilde{r}=a(t) r, x^{0}=t, x^{1}=r$, the two dimensional metric $h_{\mu \nu}=\operatorname{diag}\left(-1, a^{2} /\left(1-k r^{2}\right)\right)$. Here $k$ denotes the curvature of space with $k=0,1,-1$ corresponding to open, flat, and closed universes, respectively. The dynamical apparent horizon, a marginally trapped surface with vanishing expansion, is determined by the relation $h^{\mu \nu} \partial_{\mu} \tilde{r} \partial_{\nu} \tilde{r}=0$. Straightforward calculation gives the apparent horizon radius for the FRW universe

$$
\tilde{r}_{A}=\frac{1}{\sqrt{H^{2}+k / a^{2}}}
$$

The associated temperature with the apparent horizon can be defined as $T=\kappa / 2 \pi$, where $\kappa$ is the surface gravity $\kappa=\frac{1}{\sqrt{-h}} \partial_{\mu}\left(\sqrt{-h} h^{\mu \nu} \partial_{\mu \nu} \tilde{r}\right)$. Then one can easily show that the surface gravity at the apparent horizon of FRW universe can be written as

$$
\kappa=-\frac{1}{\tilde{r}_{A}}\left(1-\frac{\dot{\tilde{r}}_{A}}{2 H \tilde{r}_{A}}\right) .
$$

When $\dot{\tilde{r}}_{A} \leq 2 H \tilde{r}_{A}$, the surface gravity $\kappa \leq 0$, which leads the temperature $T \leq 0$ if one defines the temperature of the apparent horizon as $T=\kappa / 2 \pi$. Physically it is not easy to accept the negative 
temperature, the temperature on the apparent horizon should be defined as $T=|\kappa| / 2 \pi$. Recently, the connection between temperature on the apparent horizon and the Hawking radiation has been considered in [23], which gives more solid physical implication of the temperature associated with the apparent horizon.

Suppose the matter source in the FRW universe is a perfect fluid with stress-energy tensor

$$
T_{\mu \nu}=(\rho+p) u_{\mu} u_{\nu}+p g_{\mu \nu}
$$

where $\rho$ and $p$ are the energy density and pressure, respectively. The energy conservation law then leads to

$$
\dot{\rho}+3 H(\rho+p)=0
$$

where $H=\dot{a} / a$ is the Hubble parameter. Following [24], we define the work density as

$$
W=-\frac{1}{2} T^{\mu \nu} h_{\mu \nu}
$$

In our case it becomes

$$
W=\frac{1}{2}(\rho-p)
$$

The work density term is regarded as the work done by the change of the apparent horizon. Assuming the first law of thermodynamics on the apparent horizon is satisfied and has the form

$$
d E=T_{h} d S_{h}+W d V
$$

where $S_{h}$ is the quantum corrected entropy associated with the apparent horizon which has the form (1). One can see that in Eq. (9), the work density is replaced with the negative pressure if we compare with the standard first law of thermodynamics, $d E=T d S-p d V$. For a pure de Sitter space, $\rho=-p$, then the work term reduces to the standard $-p d V$ and we obtain exactly the standard first law of thermodynamics.

We also assume $E=\rho V$ is the total energy content of the universe inside a 3 -sphere of radius $\tilde{r}_{A}$, where $V=\frac{4 \pi}{3} \tilde{r}_{A}^{3}$ is the volume enveloped by 3 -dimensional sphere with the area of apparent horizon $A=4 \pi \tilde{r}_{A}^{2}$. Taking differential form of the relation $E=\rho \frac{4 \pi}{3} \tilde{r}_{A}^{3}$ for the total matter and energy inside the apparent horizon, we get

$$
d E=4 \pi \tilde{r}_{A}^{2} \rho d \tilde{r}_{A}+\frac{4 \pi}{3} \tilde{r}_{A}^{3} \dot{\rho} d t .
$$

Using the continuity equation (6), we obtain

$$
d E=4 \pi \tilde{r}_{A}^{2} \rho d \tilde{r}_{A}-4 \pi H \tilde{r}_{A}^{3}(\rho+p) d t .
$$


Taking differential form of the corrected entropy (1), we have

$$
d S_{h}=\frac{2 \pi \tilde{r}_{A}}{G}\left[1-\frac{\alpha G}{\pi \tilde{r}_{A}^{2}}-\frac{\beta G^{2}}{\pi^{2} \tilde{r}_{A}^{4}}\right] d \tilde{r}_{A} .
$$

Inserting Eqs. (8), (11) and (12) in the first law (9) and using the relation between temperature and surface gravity, we can get the differential form of the modified Friedmann equation

$$
\frac{1}{4 \pi G} \frac{d \tilde{r}_{A}}{\tilde{r}_{A}^{3}}\left[1-\frac{\alpha G}{\pi \tilde{r}_{A}^{2}}-\frac{\beta G^{2}}{\pi^{2} \tilde{r}_{A}^{4}}\right]=H(\rho+p) d t .
$$

Using the continuty equation (6), we can rewrite it as

$$
\frac{-2 d \tilde{r}_{A}}{\tilde{r}_{A}^{3}}\left[1-\frac{\alpha G}{\pi \tilde{r}_{A}^{2}}-\frac{\beta G^{2}}{\pi^{2} \tilde{r}_{A}^{4}}\right]=\frac{8 \pi G}{3} d \rho
$$

Integrating (14) yields

$$
\frac{1}{\tilde{r}_{A}^{2}}-\frac{\alpha G}{2 \pi \tilde{r}_{A}^{4}}-\frac{\beta G^{2}}{3 \pi^{2} \tilde{r}_{A}^{6}}=\frac{8 \pi G}{3} \rho,
$$

where an integration constant, which is just the cosmological constant, has been absorbed into the energy density $\rho$. Substituting $\tilde{r}_{A}$ from Eq.(3) we obtain entropy-corrected Friedmann equation

$$
H^{2}+\frac{k}{a^{2}}-\frac{\alpha G}{2 \pi}\left(H^{2}+\frac{k}{a^{2}}\right)^{2}-\frac{\beta G^{2}}{3 \pi^{2}}\left(H^{2}+\frac{k}{a^{2}}\right)^{3}=\frac{8 \pi G}{3} \rho
$$

In this way we derive the entropy-corrected Friedmann equation by starting from the first law of thermodynamics, $d E=T_{h} d S_{h}+W d V$, at apparent horizon of a FRW universe, and assuming that the associated entropy with apparent horizon has a quantum corrected relation (1). In the absence of the correction terms $(\alpha=0=\beta)$, one recovers the well-known Friedmann equation in standard cosmology. Since the last two terms in Eq. (16) can be comparable to the first term only when $a$ is very small, the corrections make sense only at early stage of the universe where $a \rightarrow 0$. When the universe becomes large, the entropy-corrected Friedmann equation reduces to the standard Friedmann equation.

It is important to note that in the literature many different modifications of entropy and therefore of Friedmann equations are studied in classical modified gravity theories. For example, in 25] the modified gravity with $\ln R$ or $R^{-n}$ terms which grow at small curvature was discussed. It was shown [25] that such a model may eliminate the need for dark energy and may provide the current cosmic acceleration. It was also demonstrated that $R^{2}$ terms are important not only for early time inflation but also to avoid the instabilities and the linear growth of the gravitational force. Thus, modified gravity with $R^{2}$ term seems to be viable classical theory. It was also argued in [17, 26] that the modified gravity where some arbitrary function of Gauss-Bonnet term is added 
to Einstein action can explain the dark energy dominated universe. It was shown that such theory may pass solar system tests and can describe the most interesting features of late-time cosmology such as the transition from deceleration to acceleration, crossing the phantom divide, current acceleration with effective (cosmological constant, quintessence or phantom) equation of state of the universe. In [27] the modification of the Friedmann equations which may be caused by $f(R)$ gravity, string-inspired scalar-Gauss-Bonnet, modified Gauss-Bonnet theories, and ideal fluid with the inhomogeneous equation of state. It was demonstrated [27] that the history of the expansion of the universe can be reconstructed through such a universal formulation. Further investigations on the cosmological implications of the modified theory of gravity have been carried out in [28].

It is also worth mentioning that Eq. (16) is in complete agreement with the result of [29]. However, our derivation is quite different from [29]. Let us stress the difference between here and 29]. First of all, the authors of [29] have derived modified Friedmann equations by applying the first law of thermodynamics, $T d S=-d E$, to the apparent horizon of a FRW universe with the assumption that the apparent horizon has temperature $T=1 / 2 \pi \tilde{r}_{A}$ and corrected-entropy like (1). It is worthy to note that the notation $d E$ in [29] is quite different from the same we used in the present work. In [29], $-d E$ is actually just the heat flux $\delta Q$ in [1] crossing the apparent horizon within an infinitesimal internal of time $d t$. But, here $d E$ is change in the the matter energy inside the apparent horizon. Besides, in [29] the apparent horizon radius $\tilde{r}_{A}$ has been assumed to be fixed. Thus, the temperature of apparent horizon can be approximated to $T=1 / 2 \pi \tilde{r}_{A}$ and there is no the term of volume change in it. But, here, we have used the matter energy $E$ inside the apparent horizon and the apparent horizon radius changes with time. This is the reason why we have included the term $W d V$ in the first law (9). Indeed, the term $4 \pi \tilde{r}_{A}^{2} \rho d \tilde{r}_{A}$ in Eq. (11) contributes to the work term, while this term is absent in $d E$ of [29]. This is consistent with the fact that in thermodynamics the work is done when the volume of the system is changed. We have assumed that $d \tilde{r}_{A}$ is the infinitesimal change in the radius of the apparent horizon in a small time interval $d t$ which causes a small change $d V$ of volume inside the apparent horizon. Since the matter energy $E$ is directly related to the radius of the apparent horizon, therefore, the change of apparent horizon radius will change the energy $d E$ inside the apparent horizon.

\section{GENERALIZED SECOND LAW OF THERMODYNAMICS}

In this section we turn to investigate the validity of the generalized second law of thermodynamics in a region enclosed by the apparent horizon. Differentiating Eq. (15) with respect to the 
cosmic time and using Eq. (마) we get

$$
\frac{-2 \tilde{r}_{A}}{\tilde{r}_{A}^{3}}\left[1-\frac{\alpha G}{\pi \tilde{r}_{A}^{2}}-\frac{\beta G^{2}}{\pi^{2} \tilde{r}_{A}^{4}}\right]=-8 \pi G H(\rho+p) .
$$

Solving for $\tilde{r}_{A}$ we find

$$
\tilde{r}_{A}=4 \pi G H \tilde{r}_{A}^{3}(\rho+p)\left[1-\frac{\alpha G}{\pi \tilde{r}_{A}^{2}}-\frac{\beta G^{2}}{\pi^{2} \tilde{r}_{A}^{4}}\right]^{-1} .
$$

One can see from the above equation that $\dot{\tilde{r}}_{A}>0$ provided the dominant energy condition, $\rho+p>0$, holds. Let us now turn to find out $T_{h} \dot{S}_{h}$ :

$$
T_{h} \dot{S}_{h}=\frac{1}{2 \pi \tilde{r}_{A}}\left(1-\frac{\dot{\tilde{r}}_{A}}{2 H \tilde{r}_{A}}\right) \frac{d}{d t}\left(\frac{A}{4 G}-\alpha \ln \frac{A}{4 G}+\beta \frac{4 G}{A}\right) .
$$

After some simplification and using Eq. (18) we obtain

$$
T_{h} \dot{S}_{h}=4 \pi H \tilde{r}_{A}^{3}(\rho+p)\left(1-\frac{\dot{\tilde{r}}_{A}}{2 H \tilde{r}_{A}}\right)
$$

In the accelerating universe the dominant energy condition may violate, $\rho+p<0$, indicating that the second law of thermodynamics,$\dot{S}_{h} \geq 0$, does not hold. However, as we will see below the generalized second law of thermodynamics, $\dot{S}_{h}+\dot{S}_{m} \geq 0$, is still fulfilled throughout the history of the universe. From the Gibbs equation we have [30]

$$
T_{m} d S_{m}=d(\rho V)+p d V=V d \rho+(\rho+p) d V,
$$

where $T_{m}$ and $S_{m}$ are, respectively, the temperature and the entropy of the matter fields inside the apparent horizon. We limit ourselves to the assumption that the thermal system bounded by the apparent horizon remains in equilibrium so that the temperature of the system must be uniform and the same as the temperature of its boundary. This requires that the temperature $T_{m}$ of the energy inside the apparent horizon should be in equilibrium with the temperature $T_{h}$ associated with the apparent horizon, so we have $T_{m}=T_{h}[30]$. This expression holds in the local equilibrium hypothesis. If the temperature of the fluid differs much from that of the horizon, there will be spontaneous heat flow between the horizon and the fluid and the local equilibrium hypothesis will no longer hold. Therefore from the Gibbs equation (21) we can obtain

$$
T_{h} \dot{S}_{m}=4 \pi \tilde{r}_{A}^{2} \dot{\tilde{r}}_{A}(\rho+p)-4 \pi \tilde{r}_{A}^{3} H(\rho+p) .
$$

To check the generalized second law of thermodynamics, we have to examine the evolution of the total entropy $S_{h}+S_{m}$. Adding equations (20) and (22), we get

$$
T_{h}\left(\dot{S}_{h}+\dot{S}_{m}\right)=2 \pi \tilde{r}_{A}^{2}(\rho+p) \dot{\tilde{r}}_{A}=\frac{A}{2}(\rho+p) \dot{\tilde{r}}_{A} .
$$


where $A$ is the apparent horizon area. Substituting $\dot{\tilde{r}}_{A}$ from Eq. (18) into (23) we find

$$
T_{h}\left(\dot{S}_{h}+\dot{S}_{m}\right)=2 \pi G A H \tilde{r}_{A}^{3}(\rho+p)^{2}\left[1-\frac{\alpha G}{\pi \tilde{r}_{A}^{2}}-\frac{\beta G^{2}}{\pi^{2} \tilde{r}_{A}^{4}}\right]^{-1} .
$$

It is important to note that the expression in the bracket of Eq. (24) is positive at the present time, i.e.,

$$
\left[1-\frac{\alpha G}{\pi \tilde{r}_{A}^{2}}-\frac{\beta G^{2}}{\pi^{2} \tilde{r}_{A}^{4}}\right]>0
$$

This is due to the fact that at the present time $\tilde{r}_{A} \gg 1$ while $\alpha \sim O(1), \beta \sim O(1)$ and $G \sim 10^{-11}$, thus $\frac{\alpha G}{\pi \tilde{r}_{A}^{2}} \ll 1$ and $\frac{\beta G^{2}}{\pi^{2} \tilde{r}_{A}^{4}} \ll 1$. At the early time where $\tilde{r}_{A} \rightarrow 0$ the generalized second law of thermodynamics may be violated but in that case the local equilibrium hypothesis is failed too. Besides, from the physical point of view, the effect of the correction terms on the entropy should be less than uncorrected term. Thus, the second and third terms on the right hand side of Eqs. (1) and (12) should be much smaller than the first term, otherwise these terms cannot be regarded as the correction terms. For all above reasons we can expand the right hand side of Eq. (24), up to the linear order of $\alpha$ and $\beta$,

$$
T_{h}\left(\dot{S}_{h}+\dot{S}_{m}\right)=2 \pi G A H \tilde{r}_{A}^{3}(\rho+p)^{2}\left[1+\frac{\alpha G}{\pi \tilde{r}_{A}^{2}}+\frac{\beta G^{2}}{\pi^{2} \tilde{r}_{A}^{4}}\right] .
$$

The right hand side of the above equation cannot be negative throughout the history of the universe, which means that $\dot{S}_{h}+\dot{S}_{m} \geq 0$ always holds. This indicates that for a universe with any spacial curvature the generalized second law of thermodynamics is fulfilled in a region enclosed by the apparent horizon.

\section{CONCLUSIONS}

In summary, applying the first law of thermodynamics, $d E=T_{h} d S_{h}+W d V$, to apparent horizon of a FRW universe with any spatial curvature and assuming that the apparent horizon has temperature $T=\frac{1}{2 \pi \tilde{r}_{A}}\left(1-\frac{\dot{\tilde{r}}_{A}}{2 H \tilde{r}_{A}}\right)$, and a quantum corrected entropy-area relation, $S_{h}=$ $\frac{A}{4 G}-\alpha \ln \frac{A}{4 G}+\beta \frac{4 G}{A}$, we are able to derive modified Friedmann equations governing the dynamical evolution of the universe. We have also investigated the validity of the generalized second law of thermodynamics for the FRW universe with any spatial curvature. We have shown that, when thermal system bounded by the apparent horizon remains in equilibrium with its boundary such that $T_{m}=T_{h}$, the generalized second law of thermodynamics is fulfilled in a region enclosed by the apparent horizon. The validity of the generalized second law of thermodynamics for quantum 
corrected entropy area relation further supports the thermodynamical interpretation of gravity and provides more confidence on the profound connection between gravity and thermodynamics.

It is worth noting that although we derived modified Friedmann equations corresponding to the corrected entropy-area relation (1) by applying the first law of thermodynamics to apparent horizon, it would be of great interest to see whether one is able to get modified Einstein field equation by following Jacobson argument [1]. This study is of great interest and further shows that given a thermodynamical relation between entropy and geometry, one is able to derive corresponding modified Einstein field equation, showing an interesting connection between them.

Finally, we would like to mention that the higher order terms of $\left(H^{2}+k / a^{2}\right)$ in the modified Friedmann equations (16) only becomes important at early time of the universe. They may influence the number of e-folds of inflation, or they may give corrected upper bound on the number of e-folds following the holographic principle. These should be examined carefully. Eq. (16) does not look to influence the late time cosmology. The detail of this study will be addressed elsewhere.

\section{Acknowledgments}

I thank the anonymous referees for constructive comments. I am also grateful to Prof. B. Wang for helpful discussions. This work has been supported by Research Institute for Astronomy and Astrophysics of Maragha, Iran.

[1] T. Jacobson, Phys. Rev. Lett. 75, 1260 (1995).

[2] C. Eling, R. Guedens, and T. Jacobson, Phys. Rev. Lett. 96, 121301 (2006).

[3] M. Akbar and R. G. Cai, Phys. Lett. B 635, 7 (2006) ;

M. Akbar and R. G. Cai, Phys. Lett. B 648, 243 (2007).

[4] T. Padmanabhan, Class. Quantum Grav. 19, 5387 (2002);

T. Padmanabhan, Phys. Rept. 406, 49 (2005);

T. Padmanabhan, gr-qc/0606061;

A. Paranjape, S. Sarkar and T. Padmanabhan, Phys. Rev. D 74, 104015 (2006);

D. Kothawala, S. Sarkar and T. Padmanabhan, gr-qc/0701002

T. Padmanabhan and A. Paranjape, gr-qc/0701003.

[5] M. Akbar and R. G. Cai, Phys. Rev. D 75, 084003 (2007).

[6] R. G. Cai and L. M. Cao, Phys.Rev. D 75, 064008 (2007).

[7] R. G. Cai and S. P. Kim, JHEP 0502, 050 (2005). 
[8] A. V. Frolov and L. Kofman, JCAP 0305, 009 (2003);

U. K. Danielsson, Phys. Rev. D 71, 023516(2005);

R. Bousso, Phys. Rev. D 71, 064024 (2005);

G. Calcagni, JHEP 0509, 060 (2005);

U. H. Danielsson, hep-th/0411172

[9] E. Verlinde, hep-th/0008140

B. Wang, E. Abdalla and R. K. Su, Phys.Lett. B 503, 394 (2001);

B. Wang, E. Abdalla and R. K. Su, Mod. Phys. Lett. A 17, 23 (2002);

R. G. Cai and Y. S. Myung, Phys. Rev. D 67, 124021 (2003).

[10] R. G. Cai and L. M. Cao, Nucl. Phys. B 785 (2007) 135.

[11] A. Sheykhi, B. Wang and R. G. Cai, Nucl. Phys. B779 (2007)1.

[12] A. Sheykhi, B. Wang and R. G. Cai, Phys. Rev. D 76 (2007) 023515;

A. Sheykhi, JCAP 05 (2009) 019.

[13] T. Zhu and J-R. Ren, Eur. Phys. J. C 62 (2009) 413;

R-G. Cai et al, Class.Quant.Grav 26 (2009) 155018.

[14] J. Zhang, Phys. Lett. B 668 (2008) 353;

R. Banerjee and B. R. Majhi, Phys. Lett. B 662, 62 (2008);

R. Banerjee and B. R. Majhi, JHEP 0806 (2008) 095;

R. Banerjee, B. R. Majhi and S. Samanta, Phys. Rev. D 77, 124035 (2008).

[15] C. Rovelli, Phys. Rev. Lett. 77 (1996) 3288;

A. Ashtekar, J. Baez, A. Corichi, and K. Krasnov, Phys. Rev. Lett. 80 (1998) 904;

[16] S. Nojiri and S. D. Odintsov, Int. J. Mod. Phys. A 16 (2001) 3273;

J. Lidsey et. al., Phys. Lett. B 544 (2002) 337;

S. Nojiri, S. D. Odintsov, Phys. Rev. D 72 (2005) 023003;

[17] G. Cognola, E. Elizalde, S. Nojiri, S. D. Odintsov, S. Zerbini, Phys. Rev. D 73 (2006) 084007.

[18] A. Sheykhi, Phys. Rev. D 81, 104011 (2010).

[19] B. Wang, Y. Gong, E. Abdalla, Phys. Rev. D 74 (2006) 083520.

[20] J. Zhou, B. Wang, Y. Gong, E. Abdalla, Phys. Lett. B 652 (2007) 86.

[21] A. Sheykhi, B. Wang, Phys. Lett. B 678 (2009) 434;

A. Sheykhi, Class. Quantum Grav. 27 (2010) 025007;

A. Sheykhi, B. Wang, Mod. Phys. Lett. A, Vol. 25, No. 14 (2010) 1199.

[22] M. Akbar, Chin. Phys. Lett. 25, 4199 (2008);

M. Akbar, Int. J. Theor. Phys. 48, 2665 (2009).

[23] R.G. Cai, L.M. Cao, Y.P. Hu, Class. Quantum. Grav. 26155018 (2009);

R. Li, J. R. Ren, D. F. Shi, Phys. Lett. B 670 (2009) 446.

[24] S. A. Hayward, S.Mukohyana, andM. C. Ashworth, Phys. Lett. A 256, 347 (1999);

S. A. Hayward, Class. Quant. Grav. 15, 3147 (1998) gr-qc/9710089. 
[25] S. Nojiri, S. D. Odintsov Gen. Rel. Grav. 36 (2004) 1765.

[26] S. Nojiri, S. D. Odintsov, Phys. Lett. B 631 (2005) 1.

[27] K. Bamba, S. Nojiri, S. D. Odintsov, JCAP 0810 (2008) 045.

[28] S. Nojiri, S.D. Odintsov, Int. J. Geom. Meth. Mod. Phys. 4, (2007) 115;

S. Nojiri, S. D. Odintsov, Phys. Rev. D 68 (2003) 123512;

S. Capozziello, V.F. Cardone, E. Elizalde, S. Nojiri, S. D.Odintsov Phys. Rev. D 73 (2006) 043512;

S. Nojiri, S. D. Odintsov, Phys. Lett. B 639 (2006) 144;

K. Bamba, C.Q. Geng, S. Nojiri, S. D. Odintsov, Euro phys. Lett. 89, 50003 (2010).

[29] R. G. Cai, L. M. Cao and Y. P. Hu, JHEP 0808 (2008) 090.

[30] G. Izquierdo and D. Pavon, Phys. Lett. B 633 (2006) 420. 